\title{
Beyond flu: Trends in respiratory infection outbreaks in Ontario healthcare settings from 2007 to 2017, and implications for non-influenza outbreak management
}

\author{
Katherine Paphitis ${ }^{1}$, Camille Achonu ${ }^{1 *}$, Sandra Callery ${ }^{1}$, Jonathan Gubbay ${ }^{1,2}, K_{\text {Kevin Katz }}^{3}$, \\ Matthew Muller ${ }^{4}$, Herveen Sachdeva ${ }^{5}$, Bryna Warshawsky ${ }^{1,6}$, Michael Whelan ${ }^{1}$, Gary Garber ${ }^{1,7}$, \\ Michelle Murti ${ }^{1,2}$
}

\begin{abstract}
Background: Outbreaks cause significant morbidity and mortality in healthcare settings. Current testing methods can identify specific viral respiratory pathogens, yet the approach to outbreak management remains general.
\end{abstract}

Objectives: Our aim was to examine pathogen-specific trends in respiratory outbreaks, including how attack rates, case fatality rates and outbreak duration differ by pathogen between hospitals and long-term care (LTC) and retirement homes (RH) in Ontario.

Methods: Confirmed respiratory outbreaks in Ontario hospitals and LTC/RH reported between September 1, 2007, and August 31, 2017, were extracted from the integrated Public Health Information System (iPHIS). Median attack rates and outbreak duration and overall case fatality rates of pathogen-specific outbreaks were compared in both settings.

Results: Over the 10-year surveillance period, 9,870 confirmed respiratory outbreaks were reported in Ontario hospitals and LTC/RH. Influenza was responsible for most outbreaks ( $32 \%$ in $\mathrm{LTC} / \mathrm{RH}, 51 \%$ in hospitals), but these outbreaks were shorter and had lower attack rates than most non-influenza outbreaks in either setting. Human metapneumovirus, while uncommon $(<4 \%$ of outbreaks) had high case fatality rates in both settings.

Conclusion: Attack rates and case fatality rates varied by pathogen, as did outbreak duration. Development of specific outbreak management guidance that takes into account pathogen and healthcare setting may be useful to limit the burden of respiratory outbreaks.
This work is licensed under a Creative Commons Attribution 4.0 International License.

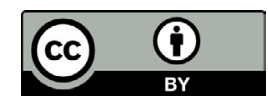

\begin{abstract}
Affiliations
1 Public Health Ontario, Toronto, ON

${ }^{2}$ University of Toronto, Toronto, $\mathrm{ON}$

${ }^{3}$ North York General Hospital, Toronto, ON

${ }^{4}$ St. Michael's Hospital, Toronto, ON

${ }^{5}$ Toronto Public Health, Toronto, ON

${ }^{6}$ Western University, London, ON

${ }^{7}$ University of Ottawa, Ottawa, ON
\end{abstract}

*Correspondence:

camille.achonu@oahpp.ca

Suggested citation: Paphitis K, Achonu C, Callery S, Gubbay J, Katz K, Muller M, Sachdeva H, Warshawsky B, Whelan M, Garber G, Murti M. Beyond flu: Trends in respiratory infection outbreaks in Ontario healthcare settings from 2007 to 2017, and implications for non-influenza outbreak management. Can Commun Dis Rep 2021;47(5/6):269-75. https://doi.org/10.14745/ccdr.v47i56a04

Keywords: disease outbreaks, health facilities, long-term care, metapneumovirus, influenza, human, morbidity

\section{Background}

Outbreaks of respiratory infections due to viral pathogens such as influenza, seasonal coronaviruses and rhinovirus occur frequently in healthcare institutions where there are multiple close contacts and an increased risk of complications due to age, underlying illness or other factors $(1,2)$. In addition, respiratory outbreaks place a large burden on the healthcare system each season, with a confirmed outbreak in a healthcare setting having implications for patient or resident admissions and transfers, as well as for staff placements within facilities.

In Ontario, respiratory infection outbreaks in institutions [including long-term care homes (LTC) and retirement homes $(\mathrm{RH})]$ and public hospitals are required to be reported to local public health units for monitoring and outbreak management 
guidance (3-5). Most respiratory infection outbreak (referred to as a "respiratory outbreak") guidance in Ontario focuses on general infection prevention and control measures using influenza as the model $(6,7)$.

Given the availability of specific interventions to prevent influenza transmission during outbreaks (e.g. antiviral prophylaxis), laboratory testing for respiratory outbreaks in Ontario has primarily focused on influenza identification. Prior to the 2009 influenza A [(H1N1) pdm09] pandemic, the primary test methods for respiratory pathogens were viral culture and rapid influenza detection tests (RIDTs). Nasopharyngeal swabs collected from LTC/RH residents are typically submitted to the provincial public health laboratory for testing, while hospitals may submit outbreak specimens to the public health laboratory or to their own laboratory. Changes in testing capabilities at the provincial public health laboratory, such as the introduction of real-time reverse transcription-polymerase chain reaction (RT-PCR) in 2009 and a multiplex respiratory viral panel (MRVP) in 2010, have allowed for more sensitive and rapid testing and identification of multiple respiratory pathogens. Similar changes have been implemented in hospital and community laboratories in Ontario, with $23 \%$ of these laboratories reporting performing multiplex molecular testing in 2017 (8). Enhanced ability to differentiate between causative pathogens allows for an opportunity to tailor infection prevention and control measures to a specific pathogen and may reduce the need for unnecessary or overly restrictive control measures in certain situations.

The aim of this study was to investigate and describe attack rates, outbreak durations and case fatality rates associated with respiratory outbreak pathogens and how these differ between hospital and LTC/RH settings.

\section{Methods}

In Ontario, respiratory outbreaks reported by institutions to local public health units are entered into the integrated Public Health Information System (iPHIS). In this study, we only analyzed outbreaks meeting the provincial definition for a confirmed respiratory outbreak, that is, two cases of acute respiratory infection within 48 hours and with a common epidemiological link, at least one of which is laboratory confirmed; or three cases of acute respiratory infection within 48 hours, with a common epidemiological link and without laboratory confirmation (9). Outbreaks occurring in LTC or RH were combined into a single category (LTC/RH). Reported information used in our analyses included institution identifiers; dates of illness onset in the first and last identified cases; outbreak report dates; laboratory findings; total patient/resident cases; total patients/residents at risk; and deaths among outbreak cases $(3,4)$.

Confirmed outbreaks reported in institutions between September 1, 2007, and August 31, 2017, were extracted from iPHIS. Respiratory outbreak seasons were defined as September 1 to August 31 of the following year based on the date the outbreak was reported or the date the outbreak was entered in iPHIS if the report date was missing $(n=67)$. Outbreak duration was defined as the period in days from the date of symptom onset for the first identified case to that of the last identified case. Outbreaks where the onset date in the first or last reported case was missing or was improbable were excluded from analyses involving duration $(n=704)$.

Attack rates were calculated as the number of patient/resident cases divided by the total number of patients/residents at risk within the affected area (e.g. entire facility, floor or unit). Case fatality rates were calculated as the number of outbreak-related deaths divided by the total number of resident/patient cases, and multiplied by 100 . Outbreaks with improbable values, such as attack rates or case fatality rates greater than $100 \%$, were excluded from attack rate and case fatality rate analyses $(n=341)$.

Outbreaks due to enterovirus, rhinovirus or enterovirus/rhinovirus were collapsed into a single category, "entero/rhinovirus," and influenza viruses ( $A, B$, or $A$ and $B$ ) were collapsed into a single category, "influenza." Outbreaks where more than one pathogen was detected were classified as "multiple." Outbreaks due to adenovirus were excluded from some analyses due to their low number $(n=5)$. Outbreaks where no specific pathogen was identified were classified as "unknown."

Analyses were performed in SAS 9.4 (SAS Institute Inc., Cary, North Carolina, United States). For statistical significance, differences in median outbreak duration and median resident/patient attack rates between hospital and LTC/RH settings for individual pathogens were assessed using the Wilcoxon-Mann-Whitney test for a difference in medians. Overall, pathogen-specific case fatality rates in either setting were compared using the Pearson Chi-square test or the Fisher exact test. For all analyses, the statistical significance level was $5 \%(\alpha=0.05)$.

Research ethics committee approval was not required for this project as the activities described here are considered routine surveillance (10).

\section{Results}

As of December 31, 2019, there were 151 hospitals and $1,392 \mathrm{LTC} / \mathrm{RH}$ operational in Ontario (11). Over the 10 years of surveillance, 9,870 respiratory outbreaks occurred in Ontario hospitals and LTC/RH (Table 1). Most of the outbreaks occurred in LTC/RH (92.0\%); 1,210 LTC/RH reported one or more respiratory outbreaks during the study period (86.9\%). The number of outbreaks reported by individual LTC/RHs ranged from 1 to 139 with a median of five outbreaks per home. In contrast, 93 hospitals reported one or more respiratory 
outbreaks during the study period (61.6\%). The number of outbreaks reported by a single hospital ranged from 1 to 133 over the 10-year study period, with a median of two outbreaks per hospital.

Table 1: Confirmed respiratory outbreaks in institutional healthcare settings, by pathogen, Ontario, 2007/2008 to $2016 / 2017(n=9,870)$

\begin{tabular}{|c|c|c|c|c|c|c|}
\hline \multirow{3}{*}{ Pathogen } & \multicolumn{6}{|c|}{$\begin{array}{l}\text { Number and proportion of outbreaks in } \\
\text { institutional healthcare settings }\end{array}$} \\
\hline & \multicolumn{2}{|c|}{ Hospitals } & \multicolumn{2}{|c|}{ LTC/RHs } & \multicolumn{2}{|c|}{ Total } \\
\hline & $\mathbf{n}$ & $\%$ & n & $\%$ & $\mathbf{n}$ & $\%$ \\
\hline Adenovirus & 1 & 0.1 & 4 & 0.0 & 5 & 0.1 \\
\hline Coronavirus & 17 & 2.2 & 499 & 5.5 & 516 & 5.2 \\
\hline Entero/rhinovirus & 87 & 11.1 & 1,654 & 18.2 & 1,741 & 17.6 \\
\hline Influenza & 401 & 51.0 & 2,900 & 31.9 & 3,301 & 33.4 \\
\hline hMPV & 25 & 3.2 & 357 & 3.9 & 382 & 3.9 \\
\hline $\begin{array}{l}\text { Parainfluenza } \\
\text { virus }\end{array}$ & 54 & 6.9 & 482 & 5.3 & 536 & 5.4 \\
\hline RSV & 70 & 8.9 & 560 & 6.2 & 630 & 6.4 \\
\hline $\begin{array}{l}\text { Multiple } \\
\text { pathogens }\end{array}$ & 77 & 9.8 & 1,085 & 11.9 & 1,162 & 11.8 \\
\hline $\begin{array}{l}\text { Unknown } \\
\text { pathogens }\end{array}$ & 54 & 6.9 & 1,543 & 17.0 & 1,597 & 16.2 \\
\hline Total & 786 & 100.0 & 9,084 & 100.0 & 9,870 & 100.0 \\
\hline
\end{tabular}

Abbreviations: hMPV, human metapneumovirus; LTC, long-term care; $\mathrm{RH}$, retirement home; RSV, respiratory syncytial virus

Influenza virus was the most commonly reported pathogen in either setting (31.9\% LTC/RH; 51.0\% hospital). Outbreaks due to coronavirus, entero/rhinovirus and multiple pathogens increased over the study period, while the incidence of outbreaks due to an unknown pathogen decreased from 2008/2009 to 2009/2010 and stabilized in recent years (Figure 1). In both hospital and LTC/RH settings, after 2009-2010, the number of influenza outbreaks demonstrated a cyclical pattern, with a higher number of influenza outbreaks every second year, compared to previous and subsequent years (Figure 1).

\section{Attack rates}

Median attack rates for each pathogen were generally higher

Figure 1: Confirmed respiratory outbreaks in institutional healthcare settings by pathogen and season, Ontario, 2007/2008 to $2016 / 2017$ ( $n=9,870)$

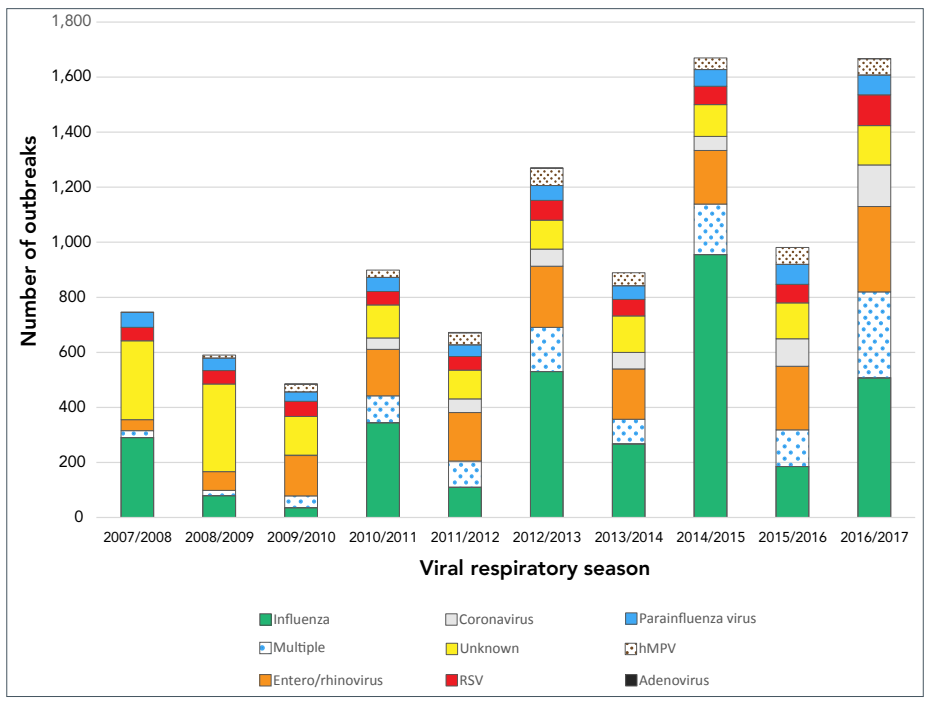

Abbreviations: hMPV, human metapneumovirus; RSV, respiratory syncytial virus

in hospitals than in LTC/RH and were highest in hospitals for outbreaks due to coronaviruses $(22.5 \%)$ and parainfluenza virus (22.0\%), and highest in LTC/RH for outbreaks due to human metapneumovirus (hMPV) (18.0\%) (Table 2). The difference in median attack rate between LTC/RH and hospitals was significantly different for outbreaks due to entero/rhinovirus, hMPV, parainfluenza virus, multiple pathogens and where the causative pathogen was unknown (Table 2).

Table 2: Median attack rates and outbreak duration for respiratory outbreaks in institutional healthcare settings, by pathogen, Ontario, 2007/2008 to 2016/2017a

\begin{tabular}{|c|c|c|c|c|c|c|c|c|c|c|}
\hline \multirow{2}{*}{ Pathogen } & \multicolumn{5}{|c|}{ Median attack rate $(\%)$} & \multicolumn{5}{|c|}{ Median outbreak duration (days) } \\
\hline & Hospital & IQR $^{\mathrm{b}}$ & LTC/RH & IQR $^{b}$ & $p$-value & Hospital & IQR $^{\mathrm{b}}$ & LTC/RH & IQR $^{b}$ & $p$-value \\
\hline Coronaviruses & 22.5 & $10.5-33.5$ & 16.0 & $10.0-23.0$ & 0.284 & 7.0 & $4.0-12.0$ & 9.0 & $5.0-14.0$ & 0.283 \\
\hline Entero/rhinovirus & 21.0 & $14.0-34.0$ & 16.0 & $10.0-24.0$ & $<0.001^{c}$ & 8.0 & $4.0-12.0$ & 8.0 & $5.0-13.0$ & 0.394 \\
\hline Influenza & 16.0 & $10.0-24.0$ & 15.0 & $9.0-24.0$ & 0.287 & 5.0 & $3.0-8.0$ & 8.0 & $5.0-12.0$ & $<0.001^{c}$ \\
\hline hMPV & 21.0 & $15.0-38.0$ & 18.0 & $11.0-27.0$ & $0.011^{c}$ & 10.0 & $5.0-14.0$ & 11.0 & $6.0-17.0$ & 0.163 \\
\hline Multiple & 20.5 & $14.0-33.0$ & 16.0 & $10.0-25.5$ & $0.001^{c}$ & 13.0 & $6.0-20.0$ & 12.0 & $7.0-19.0$ & 0.879 \\
\hline Parainfluenza virus & 22.0 & $15.0-37.0$ & 17.0 & $11.0-25.0$ & $0.001^{c}$ & 9.5 & $4.5-16.0$ & 10.0 & $5.0-16.0$ & 0.516 \\
\hline RSV & 18.0 & $11.0-28.0$ & 17.0 & $11.0-27.0$ & 0.461 & 10.0 & $5.0-16.0$ & 11.5 & $6.0-18.0$ & 0.081 \\
\hline Unknown pathogens & 21.0 & $13.0-32.0$ & 15.0 & $9.0-23.0$ & $0.001^{c}$ & 7.0 & $3.0-13.0$ & 8.0 & $4.0-13.0$ & 0.405 \\
\hline
\end{tabular}

Abbreviations: hMPV, human metapneumovirus; IQR, interquartile range; LTC, long-term care; RH, retirement home; RSV, respiratory syncytial virus

a Outbreaks due to adenovirus excluded due to low counts ( $<5$ outbreaks in either setting)

Interquartile range from the $25^{\text {th }}$ to $75^{\text {th }}$ percentile for each median value

c Significant at $P<0.05$ 


\section{Outbreak duration}

Outbreak duration ranged from 0 to 105 days in hospitals and from 0 to 122 days in LTC/RH. The difference in median outbreak duration between $\mathrm{LTC} / \mathrm{RH}$ and hospital settings was only significantly different for outbreaks due to influenza (8.0 days in LTC/RH versus 5.0 days in hospitals; $P<0.001$ ) (Table 2 ).

\section{Case fatality rates}

Most outbreaks resulted in no deaths, and overall pathogen-specific case fatality rates in either setting were relatively low (Table 3 ). Influenza had the highest case fatality rate in LTC/RH, while hMPV had the highest case fatality rate in hospitals (Table 3 ). The lowest case fatality rates were observed in entero/rhinovirus and coronavirus outbreaks in hospital and $\mathrm{LTC} / \mathrm{RH}$ settings, respectively (Table 3 ). There was a significant $(P=0.003)$ difference in overall influenza case fatality rates between hospital (2.35\%) and LTC/RH (3.54\%) settings, and for those due to unknown pathogens (Table 3 ).

Table 3: Comparison of case fatality rates for respiratory outbreaks in institutional healthcare settings, by pathogen, Ontario, $2007 / 2008$ to $2016 / 2017(n=9,844)^{\text {a }}$

\begin{tabular}{|c|c|c|c|c|c|}
\hline \multirow[b]{2}{*}{ Pathogen ${ }^{a}$} & \multicolumn{2}{|c|}{ Hospital } & \multicolumn{2}{|c|}{ LTC/RH } & \multirow[b]{2}{*}{$p$-value } \\
\hline & $\begin{array}{c}\text { Case } \\
\text { fatality } \\
\text { rate }(\%)\end{array}$ & $\begin{array}{l}\text { Number } \\
\text { of cases } \\
\text { reported } \\
\text { (n) }\end{array}$ & $\begin{array}{c}\text { Case } \\
\text { fatality } \\
\text { rate }(\%)\end{array}$ & $\begin{array}{l}\text { Number } \\
\text { of cases } \\
\text { reported } \\
\text { (n) }\end{array}$ & \\
\hline
\end{tabular}

\begin{tabular}{|l|r|r|r|r|r|}
\hline Coronaviruses & 0.88 & 113 & 0.96 & 5,815 & 1.000 \\
\hline $\begin{array}{l}\text { Entero/ } \\
\text { rhinovirus }\end{array}$ & 0.82 & 612 & 1.62 & 20,069 & 0.117 \\
\hline Influenza & 2.35 & 2,296 & 3.54 & 41,125 & $0.003^{\mathrm{b}}$ \\
\hline hMPV & 3.43 & 175 & 3.25 & 4,649 & 0.895 \\
\hline $\begin{array}{l}\text { Parainfluenza } \\
\text { virus }\end{array}$ & 2.78 & 431 & 2.12 & 6,047 & 0.357 \\
\hline RSV & 2.65 & 415 & 2.39 & 7,628 & 0.732 \\
\hline $\begin{array}{l}\text { Multiple } \\
\text { pathogens }\end{array}$ & 2.32 & 732 & 3.19 & 17,912 & 0.189 \\
\hline $\begin{array}{l}\text { Unknown } \\
\text { pathogens }\end{array}$ & 3.39 & 501 & 1.76 & 16,040 & $0.007^{\mathrm{b}}$ \\
\hline Total & 2.33 & 5,275 & 2.64 & 119,285 & $\mathrm{~N} / \mathrm{A}$ \\
\hline
\end{tabular}

Abbreviations: hMPV, human metapneumovirus; LTC, long-term care; N/A, not applicable; $\mathrm{RH}$, retirement home; RSV, respiratory syncytial virus

Outbreaks due to adenovirus excluded due to low counts ( $<5$ outbreaks in either setting) ${ }^{b}$ Significant at $P<0.05$

\section{Discussion}

Outbreaks of respiratory infection can cause significant morbidity and disruption to residents and patients in healthcare institutions each year. This study found that $61.6 \%$ of hospitals and $86.9 \%$ of $\mathrm{LTC} / \mathrm{RH}$ had one or more outbreaks over the surveillance period, with LTC/RH generally having a higher number of outbreaks than hospitals. Compared to LTC/RH residents, hospitalized individuals with respiratory symptoms are more easily isolated from others in a single room, placed in a cohort with others with similar illness or discharged from the facility to limit their potential for transmission within the facility. Conversely, increased independent mobility of residents in LTC/RH and participation in group dining and other activities likely contribute to transmission of illness in these settings.

Differences in the number of outbreaks between facility types may also be due to differences in reporting and facility size. Changes in specimen testing over time have contributed to improving the detection of outbreaks that are not associated with influenza. The ability to identify and differentiate between non-influenza respiratory pathogens may be helpful for outbreak management and control, particularly given the varying degree to which individual pathogens are found to be responsible for outbreaks, and the associated variability in outbreak duration, attack rate and case fatality rate, as observed in this study.

Because of the nature of facility design, hospital outbreaks may be more easily restricted to smaller areas such as a ward, unit or floor, whereas an outbreak in a LTC/RH may be more likely to occur across the facility, increasing the number of residents/staff at risk of illness and reducing overall attack rates. This may have contributed to the higher attack rates in hospitals compared to LTC/RH settings for outbreaks due to each viral respiratory pathogen examined in this study.

In addition, differences in the proportion of respiratory outbreaks due to unknown pathogens may be attributed to different testing algorithms used in hospitals and LTC/RH facilities as LTC/RH primarily rely on the public health laboratory for testing while many hospitals conduct their own testing and may have different testing criteria.

While most respiratory outbreaks in both LTC/RH and hospital settings in this study were due to influenza, influenza outbreaks also had the shortest median duration of all the hospital outbreaks and one of the shortest for LTC/RH. The median duration of outbreaks due to influenza was also significantly lower in hospital settings than in LTC/RH settings. The early introduction of antivirals for both treatment and prophylaxis in influenza outbreaks is known to quickly bring outbreaks under control, shortening outbreak duration and lowering the attack rate (12-14). Adherence to existing influenza outbreak management guidance, which indicates the provision of antivirals to all patients/residents, likely contributed to the low attack rates and short outbreak duration observed in this study.

We observed that peaks of influenza outbreaks followed a biennial trend corresponding to influenza A (H3N2) dominant seasons. This is consistent with previous research that has shown that there are typically increased numbers of influenza outbreaks in hospital settings in influenza A (H3N2) dominant years (15).

Current Ontario respiratory guidance advises that most outbreaks may be declared over eight days after the onset of symptoms in the last identified patient/resident case (consistent with one incubation period plus one communicable period for influenza) (6). As influenza has the shortest incubation period of the pathogens examined in this study, management 
of all outbreaks as if these are influenza outbreaks could potentially mean that outbreak control measures are lifted too soon. Identification of a specific pathogen allows for pathogen-specific incubation and communicable periods to be taken into consideration when determining when to declare an outbreak over, ensuring that control measures remain in place for an appropriate length of time.

In this study, outbreaks due to multiple pathogens were associated with significantly higher attack rates in hospital settings than in LTC/RH. Outbreaks due to multiple pathogens were also associated with the longest median outbreak duration in both settings. This may be due to concurrently circulating pathogens or to overlapping outbreaks caused by different pathogens, increasing both the potential for illness among patients/residents and complexity of outbreak management. Where outbreaks are due to several concurrently circulating pathogens, it would be prudent to institute control measures as per the pathogen with the longest incubation and/or communicable period.

Overall, case fatality rates were significantly higher in LTC/RH settings than in hospitals for outbreaks due to influenza and unknown pathogens. Residents in LTC/RH may be at increased risk of death associated with influenza, despite the use of antiviral medications, due to older age as well as the presence of other comorbidities, increasing their risk of severe outcomes from influenza, including pneumonia and death $(16,17)$, and due to advanced directives that may preclude receiving hospital-level care. A study by luliano et al. (2018) found that influenza-associated excess mortality rates increased with age in Canada, with those aged 75 years and older having the highest influenza-associated excess mortality rates compared to younger age cohorts (17).

Outbreaks due to hMPV were associated with the highest case fatality rate in hospital settings and the second highest case fatality rate in LTC/RH. Several outbreaks due to hMPV have also been described in the literature, where these resulted in high attack rates and case fatality rates in LTC settings in the United States, highlighting the importance of testing to identify hMPV as a causative pathogen, particularly as the seasonality and symptoms associated with hMPV are similar to other respiratory pathogens $(18,19)$. Although outbreaks due to hMPV and parainfluenza virus accounted for a small proportion of outbreaks due to known pathogens in either setting $(<4 \%)$, their comparatively high median attack rates and the high overall case fatality rate for hMPV may warrant specific outbreak management guidelines, such as stricter isolation, placement in cohorts and restricting movement, which could potentially contribute to reduced attack rates and subsequent mortality in both hospital and LTC/RH settings from these pathogens. Conversely, while entero/rhinovirus were a common cause of outbreaks in both hospital and LTC/RH settings, these outbreaks were associated with generally lower attack rates, case fatality rates and outbreak duration than outbreaks associated with other pathogens. Specific outbreak management guidance for these pathogens could therefore be potentially more permissive.

\section{Strengths and limitations}

This study has several limitations. The data only represent outbreaks reported to public health units and recorded in iPHIS. Classification of the population at risk and resulting attack rates, as well as case fatality rates, are based on information reported by individual facilities to public health units and may be subject to variations in surveillance and reporting between facilities and setting type, which may have impacted comparisons between settings. In addition, the implementation of outbreak control measures may vary between settings due to contextual differences that may impact the variability in outcomes observed in this study. Laboratory testing practices in hospital settings performing their own specimen testing may vary regionally across the province, and over time as testing practices change. As current outbreak testing algorithms are hierarchical and initially focused on identifying or ruling out influenza, some outbreaks due to multiple pathogens may have been misclassified as influenza outbreaks only.

\section{Conclusion}

Given the increasing burden of respiratory outbreaks on the provincial healthcare system in Ontario and elsewhere, this study highlights potential opportunities where rapid outbreak detection, pathogen confirmation and implementation of pathogen-specific outbreak control measures may have positive implications for limiting transmission of illness and outbreak duration. Current outbreak guidance in Ontario and the United States focuses on influenza $(13,20)$.

In addition, specific guidance for hospitals, recognizing the different challenges in acute care facilities and in LTC/RH facilities, may support outbreak management practices in these settings. Several Canadian provinces have developed respiratory outbreak guidance for acute care settings and LTC/RH, including recommendations for the management of patients with specific pathogens $(21,22)$, a potential model to consider in developing locally applicable resources to guide the management of respiratory outbreaks.

While data analyzed for this study predated the collection of data on outbreaks due to severe acute respiratory syndrome coronavirus 2 (SARS-CoV-2), it is anticipated that the widespread implementation of infection prevention and control measures aimed at preventing transmission of SARS-CoV-2 in institutions during the 2020 pandemic will have had an added benefit in reducing transmission of other respiratory pathogens spread via droplet/contact. This benefit is expected to be most evident in LTC and RH, where facilities likely had less on-site expertise and human resources dedicated to infection prevention and control activities. 
This study provides a valuable comparator and baseline for future studies that aim to assess the broader impact of SARS-CoV-2 prevention and control measures in institutions.

\section{Authors' statement}

$\mathrm{KP}$ - Analyzed and interpreted the outbreak data and was a major contributor in writing the manuscript

$\mathrm{CA}$ - Analyzed and interpreted the outbreak data and was a major contributor in writing the manuscript

SC - Made substantial contributions to the conception of the work and to the revision of the manuscript

JG - Made substantial contributions to the conception of the work, interpretation of laboratory testing methods and to the revision of the manuscript

KK - Made substantial contributions to the conception of the work and to the revision of the manuscript

MM - Made substantial contributions to the conception of the work and to the revision of the manuscript

HS - Made substantial contributions to the conception of the work and to the revision of the manuscript

BW - Made substantial contributions to the interpretation of the outbreak data and to the revision of the manuscript

MW - Made substantial contributions to the conception of the work and to the revision of the manuscript

GG - Made substantial contributions to the conception of the work and to the revision of the manuscript

MM - Made substantial contributions to the conception of the work, interpretation of the outbreak data and to the revision of the manuscript

All authors read and approved the final manuscript.

The content and view expressed in this article are those of the authors and do not necessarily reflect those of the Government of Canada.

\section{Competing interests}

The authors declare that they have no competing interests.

\section{Funding}

This study did not receive any external funding. All work on this study was completed at and supported by Public Health Ontario.

\section{References}

1. Longtin J, Marchand-Austin A, Winter AL, Patel S, Eshaghi A, Jamieson F, Low DE, Gubbay JB. Rhinovirus outbreaks in long-term care facilities, Ontario, Canada. Emerg Infect Dis 2010;16(9):1463-5. DOI PubMed
2. Childs A, Zullo AR, Joyce NR, McConeghy KW, van Aalst R, Moyo P, Bosco E, Mor V, Gravenstein S. The burden of respiratory infections among older adults in long-term care: a systematic review. BMC Geriatr 2019;19(1):210. DOl PubMed

3. Health Protection and Promotion Act, R.S.O. 1990, c. H.7. Toronto (ON): Government of Ontario (accessed 2019-10-25). https://www.ontario.ca/laws/ statute/90h07

4. Ontario RR. 1990, Reg. 569: Reports. Toronto (ON): Government of Ontario; 2018 (accessed 2019-10-25). https://www.ontario.ca/laws/regulation/900569

5. Ministry of Health and Long-Term Care. Infectious diseases protocol. Appendix A: disease-specific chapters. Chapter: respiratory infection outbreaks in institutions and public hospitals. Toronto (ON): MoHLTC; 2019 (accessed 2019-10-25). http://www.health.gov.on.ca/en/pro/ programs/publichealth/oph_standards/docs/respiratory_ outbreaks_chapter.pdf

6. Ministry of Health and Long-Term Care. Control of respiratory infection outbreaks in long-term care homes. Toronto (ON): MoHLTC; 2018 (accessed 2019-10-25). http://www.health.gov.on.ca/en/pro/programs/publichealth/ oph_standards/docs/reference/RESP_Infectn_ctrl_guide_ LTC_2018_en.pdf

7. Ontario Hospital Association and the Ontario Medical Association Joint Communicable Diseases Surveillance Protocols Committee. Influenza surveillance protocol for Ontario hospitals. Toronto (ON): Ontario Hospital Association; 2018 (accessed 2019-10-25). https://www.oha. com/Documents/Influenza\%20Protocol\%20(November\%20 2018).pdf

8. Kozak R, Kerr E, Richardson D, Vermeiren C, Poutanen SM. How modern is your microbiology laboratory? Results of the 2017 Institute for Quality Management in Healthcare (IQHM) patterns of practice survey. (Presentation). 2019 (accessed 2019-10-25). https://iqmh.org/Portals/0/ Docs/Resources/Poster\%20-\%20MICRO\%20-\%20SUNCPHM-826\%20How\%20Modern\%20ls\%20Your\%20Lab\%20 Oral.pdf

9. Ministry of Health and Long-Term Care. Infectious diseases protocol. Appendix B: provincial case definitions for diseases of public health significance. Disease: respiratory infection outbreaks in institutions and public hospitals. Toronto (ON): MoHLTC; 2019 (accessed 2019-10-25). http://www. health. gov.on.ca/en/pro/programs/publichealth/oph_standards/ docs/respiratory_outbreaks_cd.pdf

10. Ontario Agency for Health Protection and Promotion Act. 2007, SO 2007, c. 10, Sched. K. Toronto (ON): Government of Ontario (accessed 2019-10-25). https://www.ontario.ca/ laws/statute/07o10

11. Ontario Ministry of Health. Ministry of Long-Term Care. Ministry reports: master numbering system. Toronto (ON): MoHLTC; 2020 (accessed 2019-10-25). http://www. health. gov.on.ca/en/common/ministry/publications/reports/master_ numsys/master_numsys.aspx 
12. Ye M, Jacobs A, Khan MN, Jaipaul J, Oda J, Johnson M, Doroshenko A. Evaluation of the use of oseltamivir prophylaxis in the control of influenza outbreaks in long-term care facilities in Alberta, Canada: a retrospective provincial database analysis. BMJ Open 2016;6(7):e011686. DOI PubMed

13. Centers for Disease Control and Prevention. Prevention strategies for seasonal influenza in healthcare settings: guidelines and recommendations. Atlanta (GA): CDC: 2018 (accessed 2019-10-25). https://www.cdc.gov/flu/ professionals/infectioncontrol/healthcaresettings.htm

14. Murti M, Fung CK, Chan K, Bigham M. Duration of influenza outbreaks in long-term care facilities after antiviral prophylaxis initiation: fraser Health, British Columbia, 2014-2017. Am J Infect Control 2018;46(9):1077-9. DOI PubMed

15. Murti M, Whelan M, Friedman L, Savic J, Johnstone J, Sider D, Warshawsky B. Influenza outbreaks in Ontario hospitals, 2012-2016.

Can Commun Dis Rep 2018;44(9):201-5. DOI PubMed

16. Clay CC, Donart N, Fomukong N, Knight JB, Overheim K, Tipper J, Van Westrienen J, Hahn F, Harrod KS. Severe acute respiratory syndrome-coronavirus infection in aged nonhuman primates is associated with modulated pulmonary and systemic immune responses. Immun Ageing 2014;11(1):4. DOI PubMed

17. Iuliano AD, Roguski KM, Chang $\mathrm{HH}$, Muscatello DJ, Palekar R, Tempia S, Cohen C, Gran JM, Schanzer D, Cowling BJ, Wu P, Kyncl J, Ang LW, Park M, Redlberger-Fritz M, Yu H, Espenhain L, Krishnan A, Emukule G, van Asten L, Pereira da Silva S, Aungkulanon S, Buchholz U, Widdowson MA, Bresee JS; Global Seasonal Influenza-associated Mortality Collaborator Network. Estimates of global seasonal influenza-associated respiratory mortality: a modelling study. Lancet 2018;391(10127):1285-300. DOI PubMed
18. Boivin G, De Serres G, Hamelin ME, Côté S, Argouin M, Tremblay G, Maranda-Aubut R, Sauvageau C, Ouakki M, Boulianne N, Couture $C$. An outbreak of severe respiratory tract infection due to human metapneumovirus in a long-term care facility. Clin Infect Dis 2007;44(9):1152-8. DOl PubMed

19. Liao RS, Appelgate DM, Pelz RK. An outbreak of severe respiratory tract infection due to human metapneumovirus in a long-term care facility for the elderly in Oregon. J Clin Virol 2012;53(2):171-3. DOI PubMed

20. Centers for Disease Control and Prevention. Interim guidance for influenza outbreak management in long-term care and post-acute care facilities. Atlanta (GA): CDC: 2019 (accessed 2019-10-25). https://www.cdc.gov/flu/ professionals/infectioncontrol/ltc-facility-guidance.htm

21. AHS Outbreak Management Working Group. Guidelines for outbreak prevention, control and management in acute care and facility living sites: includes Influenza and Gastrointestinal Illness. Edmonton (AB): Alberta Health Services; 2019 (accessed 2019-10-25). https://www. albertahealthservices.ca/assets/healthinfo/hi-dis-flu-prov-hlsl. pdf

22. Alberta Health Services. IPC diseases and conditions table: recommendations for management of patients - acute care. Edmonton (AB): AHS; 2019 (accessed 2019-10-25). https:// www.albertahealthservices.ca/assets/healthinfo/ipc/hi-ipcresource-manual-main-document.pdf 